doi:10.1016/j.yrtph.2006.04.004

Copyright (c) 2006 Elsevier Inc. All rights reserved.

\title{
Hazard classification of chemicals inducing haemolytic anaemia: An EU regulatory perspective
}

\section{Andre Muller ${ }^{a}, *$, Helene Jacobsen ${ }^{b}$, Edel Healy ${ }^{c}$, Sinead McMickan ${ }^{c}$, Fréderique Istace ${ }^{d}$, Marie-Noëlle Blaude ${ }^{d}$, Peter Howden ${ }^{e}$, Helmut Fleigf, Agnes Schulte ${ }^{g}$ and (EU Working Group on Haemolytic Anaemia)}

${ }^{a}$ Rijksinstituut voor Volksgezondheid en Milieu, Antonie van Leeuwenhoeklaan 9, 3721 MA Bilthoven, The Netherlands

${ }^{b}$ Danish Institute for Food and Veterinary Research, Department of Toxicology and Risk Assessment, Moerkhoej Bygade 19, 2860 Soeborg, Denmark

'Hazardous Substances Assessment Unit, Health and Safety Authority, 10 Hogan Place, Dublin 2, Ireland

${ }^{\mathrm{d} S}$ Scientific Institute for Public Health, Division Toxicology, Wytsman 14, 1050

Brussels, Belgium

${ }^{\mathrm{e}}$ Industrial Chemicals Unit, Health and Safety Executive, 252 Magdalen House, Bootle L20 3QZ, UK

fBASF AG, Toxikologie, Ludwigshafen am Rhein, Germany

${ }^{9}$ Federal Institute for Risk Assessment, Toxicology of Chemicals, Thielallee 88-92, D-14195 Berlin, Germany

* Corresponding author.

\begin{abstract}
Haemolytic anaemia is often induced following prolonged exposure to chemical substances. Currently, under EU Council Directive 67/548/EEC, substances which induce such effects are classified as dangerous and assigned the risk phrase R48 'Danger of serious damage to health by prolonged exposure.' Whilst the general classification criteria for this endpoint are outlined in Annex VI of this Directive, they do not provide specific information to assess haemolytic anaemia. This review produced by the EU Working Group on Haemolytic Anaemia provides a toxicological assessment of haemolytic anaemia and proposes criteria that can be used in the assessment for classification of substances which induce such effects. An overview of the primary and secondary effects of haemolytic anaemia which can occur in rodent repeated dose toxicity studies is given. A detailed analysis of the toxicological significance of such effects is then performed and correlated with the general classification criteria used for this endpoint. This review intends to give guidance when carrying out an assessment for classification for this endpoint and to allow for better transparency in the decision-making process on when to classify based on the presence of haemolytic anaemia in repeated dose toxicity studies. The extended classification criteria for haemolytic anaemia outlined in this review were accepted by the EU Commission Working Group on the Classification and Labelling of Dangerous Substances in September 2004.
\end{abstract}




\section{Introduction}

Within the European Union, protection of human health and the environment from the dangerous effects of hazardous chemicals is achieved in part by classification and labelling of such chemicals. The classification has a large number of downstream consequences within EU legislation. The requirements for the classification and labelling of substances are described in Annex VI of Council Directive 67/548/EEC and its amendments (EU, 1967). For each endpoint such as carcinogenicity, irritation or acute toxicity classification criteria are provided which include dose limits and/or effect limits. Substances which induce adverse effects following prolonged exposure such as that observed in repeated dose toxicity studies at dose levels below specified limits are classified and labelled as 'Danger of serious damage to health by prolonged exposure' and assigned the risk phrase R48. The decision to classify for this endpoint depends on several factors including the severity of the effect, the reversibility of the effect, magnitude of the effect and the possibility of a serious impact on health after prolonged exposure. Further information on classification and labelling in the EU can be found at: http://ecb.jrc.it/classification-labelling/.

Haemolytic anaemia is one effect often found in repeated-dose toxicity studies. However, it is often unclear when the primary and secondary effects of haemolytic anaemia warrant classification of a substance with R48, which, in part, is due to the non-specific nature of the criteria outlined in the aforementioned Directive. This has resulted in many discussions between the member states and industry in EU Commission Working Groups on the Classification and Labelling of Dangerous Substances meetings. To resolve this problem, a working group was formed to propose specific criteria for the classification of substances inducing haemolytic anaemia with R48.

To facilitate consistent classification of substances causing haemolytic anaemia, a description of the primary and secondary effects of haemolytic anaemia is provided including a non-exhaustive review of the possible toxicological indicators of haemolytic anaemia. Classification criteria that can be used to aid in the classification of a substance for this endpoint under the EU system are proposed in Appendix A.

The proposed criteria were accepted by the EU Commission Working Group on the Classification and Labelling of Dangerous Substances in the meeting of September 2004, JRC, Ispra, Italy.

\section{Overview of anaemia}

\subsection{Anaemia}

Anaemia is usually defined as a reduction of the haemoglobin concentration, red blood cell count or packed cell volume to below normal levels. As a result, the oxygen carrying ability of the blood is reduced. The causes of anaemia are divided into failure of red cell proliferation, defective maturation of red blood cells, haemolysis, and blood loss. Exogenous agents can induce anaemia by some of these different mechanisms. Classification of chemicals that cause anemia by other mechanisms, e.g. blood loss or direct toxicity to the bone marrow resulting in decreased bone marrow production of red blood cells, are not considered in this review. 
Symptoms of anaemia, depend on three factors: the causative disorder, the degree of erythrocyte destruction and the reduction of oxygen-carrying capacity. The symptoms can vary with the severity of anaemia from decreased physical activity, fatigue, headache, faintness, increased sensitivity to cold, tinnitus, black spots before the eyes, irritability, lack of power of concentration, fever, pallor, dyspnoea, tachycardia, systolic murmurs, cyanosis, nausea, vomiting and abdominal pain to death.

Anaemia can cause serious damage to health. For example anaemic older people without clinical disease have an increased risk of mortality (Izaks et al., 1999) and there is an association between anaemia and greater physical decline (Penninx et al., 2003). There is increasing evidence that anaemia adversely affects the health and life quality of individuals (Baker and DeMaeyer, 1979). It should be kept in mind that weakness, fatigue or lassitude may accompany slowly developing chronic anaemia, in the absence of other symptoms and reduced exercise tolerance in patients.

\subsection{Haemolytic anaemia}

Haemolytic anaemia is an anaemia caused by accelerated destruction of mature red cells outside the bone marrow or a consequence of the destruction of imperfectly formed red cells. Haemolytic disorders can be classified as intracorpuscular defects, which are mainly hereditable disorders (e.g. hereditary spherocytosis, G6PD-deficiencies, thalassemia, sickle cell disease, etc.) and extracorpuscular defects (caused by e.g. infectious, chemical and physical agents and auto-antibodies). Haemolysis may be intravascular and extravascular or a combination of both.

Classical signs of haemolytic anaemia are hyperbilirubinemia often accompanied by jaundice, increased excretion of haemoglobin breakdown products (haemoglobin or haemosiderin) in the urine and stools and evidence of appropriate bone marrow erythropoietic response (reticulocytosis).

Haemolytic anaemia induced by chemical agents may be more detrimental for people with hereditable haemolytic disorders and for people with pre-existing anaemia. Vulnerable subgroups for haemolytic anaemia are individuals already low in haemoglobin status including women of menstruating age (Barr et al., 1998), children (Tapiero et al., 2001) and the elderly (Beutler, 1995).

\subsubsection{Intravascular haemolysis}

During intravascular haemolysis, the red blood cell is lysed within the general circulation so that haemoglobinemia is pronounced and hyperbilirubinemia may occur. Free haemoglobin in the circulation is complexed by haptoglobin and metabolised in the reticuloendothelial system. If the binding capacity of haptoglobin is exceeded, haemoglobin appears in the plasma where it is degraded, and the haem, which is liberated, binds to haemopexin. When the binding capacities of haptoglobin and haemopexin are saturated, free haemoglobin appears in the plasma where it is rapidly oxidized to methaemoglobin (MetHb), dissociated, and the haem binds to albumin to form methaemalbumin (Erslev and Beutler, 1995). Remaining unbound (met)haemoglobin is filtered into the primary urine and reabsorbed in the renal proximal tubules, where the iron is extracted and incorporated into haemosiderin. Haemoglobinuria occurs when the haemoglobin concentration exceeds the resorption capacity of the proximal renal tubules and is indicative of extensive intravascular haemolysis. Haemosiderin is excreted in urine but may not be 
detectable in the urine for several days after the onset of intravascular haemolysis and may persist for several days after it has terminated. Accordingly, the detection of haemosiderin in the urine is indicative of either ongoing or recent intravascular haemolysis with excess of haemoglobin filtered through the renal glomeruli and loss of haemosiderin-laden necrotic tubular cells.

\subsubsection{Extravascular haemolysis}

During extravascular haemolysis the red blood cells are destroyed in tissues with little haemoglobin escaping into the plasma, resulting in bilirubin being predominant in the plasma instead of free (met)haemoglobin. The damaged erythrocytes are prematurely removed from the circulation by macrophages, particularly those of the spleen and the bone marrow. When the degree of haemolysis is extensive, haemosiderin may be deposited in the spleen, kidney, liver, bone marrow and other organs (haemosiderosis).

\subsubsection{Iron storage (haemosiderosis)}

\subsubsection{Overview of haemosiderois}

Iron in tissues is stored either in a ferric-soluble, non-toxic form (ferritin) that can be released into circulation on demand or it can accumulate as insoluble, aggregated deposits (haemosiderin). Storage (ferritin) iron concentration is the major factor affecting the relative distribution of iron between ferritin and haemosiderin in mammals. At low storage levels, iron is stored as ferritin that is primarily taken up by hepatocytes and reticulo-endothelial macrophages of the spleen, liver and bone marrow. As the amount of cellular iron increases (iron overload), the ferritin iron is degraded to haemosiderin (Miyazaki et al., 2002) and the haemosiderin proportion increases (Smith, 1997, Emerit et al., 2001 and Zuyderhoudt et al., 1983). Haemosiderin deposition has no physiological function in iron metabolism other than to sequester iron waste within cytosolic lysosomes. In haemolytic conditions, it indicates persistent remnants of erythrophagocytosis in tissues where there is no clearance mechanism to deliver the pigment-laden phagocytes.

In healthy individuals haemoglobin degradation of senescent erythrocytes can produce small amounts of haemosiderin in the spleen that increases with age. This reflects a normal age-related lesion in both humans and animals. In healthy conditions, haemosiderin deposits are not present in organs such as the liver or the kidney.

Following substance-induced haemolysis, haemosiderin deposition can be accelerated to such levels that the accumulated pigment exceeds the normal amount of deposits in the spleen and may affect other organs as well. It has been demonstrated for many haemolytic substances that after the end of haemolysis the amount of intracellular pigment granules will not return to the same level that is expected for control animals at the same age. Accelerated haemosiderosis deposition causes an irreversible single or multi-organ iron overload as iron is not reutilized.

Similarities in haemosiderotic organs of humans and animals suggest that haemosiderin(-iron) deposition in hepatocytes and reticuloendothelial (phagocytic) cells is a common defence mechanism to enable segregation of iron. 


\subsubsection{Understanding the mode of action}

Due to the lack of an excretion mode for iron, the iron stores in healthy men and postmenopausal women increase almost linearly with age and generate a risk for oxidative stress-related diseases like arteriosclerosis, chronic inflammation disease or cancer.

There is growing evidence that excessive intracellular (haemosiderin-)iron causes oxidative stress, which leads to cellular toxicity including DNA damage and fibrosis. Increased substance-induced haemosiderosis is thought to accelerate the cell damage through the increased release of free iron and subsequent free radical production. The tissue content of haemosiderin correlates with lysosomal fragility suggesting that haemosiderin accumulation causes lysosomal disruption and hence the cell damage (Selden et al., 1980). The amount of haemosiderosis correlates with increases in lipid peroxidation products. The involvement of ironassociated oxidative damage is supported by the protective effect of antioxidants in reducing the level of iron-induced lipid peroxidation (Inan et al., 1998).

The relevance of haemosiderosis in animals as being predictive for humans is given by the pathological manifestations of haemosiderosis in clinical conditions with iron overload produced by excessive iron absorption (in genetic haemochromatosis, or high iron supply) (Fairbanks and Baldus, 1995) or parental iron loading (repeated blood transfusion) (Harada et al., 1998). Fibrosis, cirrhosis, and/or tumours have developed (Harrison's, 2001, Weinberg, 1983, Tiniakos and Williams, 1988 and Nyska et al., 2004) as secondary conditions.

\subsubsection{Haemosiderosis in the spleen}

Some recovery ${ }^{1}$ studies on haemolytic substances point to a tendency towards a slight reduction in the amount or in the incidence of haemosiderin deposits in the spleen during the recovery period indicating a minor tendency for some resolution of such deposition. This may be due to movement of haemosiderin-laden macrophages to other organs. This idea is supported by the observation that liver-derived haemosiderin-laden macrophages were found in the lungs of rats (Hertz et al., 1992).

Haemosiderosis of the spleen may be associated with fibrosis in the red pulp area or in the subcapsular/capsular regions. Commonly fibrosis can occur after exposure to the haemolytic substance for three months or more and sometimes early fibrotic foci can also be seen in 28-day studies. In general fibrotic changes are not reversible. However, occasionally some reduction in severity has been shown. In addition, fatty (cell) metamorphosis often seen within the fibrotic tissue may occur during chronic haemolysis, the underlying pathogenesis of which is unknown. Since fibrosis is a common finding after long-lasting haemolysis and haemosiderosis that does not show remission, haemosiderosis and its related findings in experimental animals are predictive for spleen toxicity in humans unless a species-specific mechanism can be demonstrated for the haemolytic substance. They thus represent irreversible adverse effects.

\subsubsection{Haemosiderosis in the liver}

In healthy conditions, hepatocytes have a significant function in iron (ferritin) storage and can take up iron from haemoglobin, haptoglobin and heme and from ferritin coming from reticulo-endothelial phagocytic (Kupffer) cells. The turnover of the iron storage pool in the liver is very low. 
Haemosiderin deposition in the liver is a pathological finding. In the case of iron overload, ferritin and haemosiderin accumulation increase in hepatocytes, bile duct epithelium and (Kupffer) cells (Matsuno et al., 1985). Iron-overloaded hepatocytes induce activation of hepatic stellate cells leading to their transformation into myofibroblastic cells with collagen formation and resulting in liver fibrosis (Parkes and Templeton, 2003). Similar liver findings as in chronic haemolysis were seen in experimental iron-overload conditions in mice (Arrezzini et al., 2003), in rats (Duellmann et al., 1992), and dogs (Sprague et al., 2003). Liver lesions such as single cell to advanced necrosis of hepatocytes, mild to marked fibrosis, lobular atrophy and biliary hypertrophy were seen with chronic iron overload.

Pathological changes associated with liver haemosiderosis in humans (e.g. due to genetic haemochromatosis) include fibrosis, liver cell degeneration/necrosis and cirrhosis (Harada et al., 1998 and Riede et al., 1989). In humans with iron overload diseases, liver iron content ( $\mathrm{mg} / \mathrm{g}$ dry weight) was found to correlate well with microscopic estimation of iron (Fairbanks and Baldus, 1995).

\subsubsection{Haemosiderosis in the kidney}

In rodents, haemosiderin deposits may occur as a pathological finding in proximal tubules, glomeruli and interstitium. The deposits are most prominent in the renal cortical tubules where they can reverse after the end of haemolysis and, depending on the severity and extent of distribution, may be fully reversible. Reduction in renal haemosiderin deposits occurs when haemosiderin-laden tubular cells become necrotic and are sloughed into the urine. After the end of exposure to the haemolytic substance, the tubular cell loss may be replaced by tubular cell regeneration and partial or full reversibility of the haemosiderin accumulation in renal tubular cells can be observed due to the deliberation of pigment-laden cells into urine. The cell death associated with haemosiderin accumulation is clearly an adverse effect, which depending on its severity, distribution, and persistence may induce secondary toxicity such as haemosiderinuria, tubular necrosis/atrophy, interstitial inflammation or fibrosis. Haemosiderin deposits in glomeruli lead to (non-reversible) glomerulosclerosis in chronic haemosiderosis (Zhou et al., 2000). Renal haemosiderosis has also been reported in humans (Leonardi and Ruol, 1960).

\subsubsection{Gall stone formation}

Regardless of the site of destruction of haemoglobin, one of the final products is bilirubin. Bilirubin is released into the blood and binds to albumin. In the liver, bilirubin is conjugated and excreted through the bile into the gastrointestinal tract, where it is converted to urobilinogen by bacterial reduction. A small fraction of urobilinogen is reabsorbed and excreted into the urine.

Substance induced haemolytic anaemia has not been observed to result in gall stone formation in laboratory animals. However, there are strong indications that increased chronic haemolysis is a predisposing factor for bilirubinate cholelithiasis in humans, which is considered as serious damage (Sickle cell disease: Diagne et al., 1999 and Bonatsos et al., 2001, Pernicious anemia: Aydogdu et al., 2001, Hereditary spherocytosis: Marchetti et al., 1998, Sandler et al., 1999 and BaderMeunier et al., 2001, Wilsons disease: Goswami et al., 2001, Haemolysis by use heart lung machine: Azemoto et al., 1996). The spleen of patients with hereditary spherocytosis is often removed. This results in a decrease of the haemolysis and a decrease in the incidence of gall stone formation when compared to patients without splenectomy (Sandler et al., 1999). This indicates that haemolytic 
anaemia induced bilirubin formation is a contributing factor to gall stone formation.

\subsection{Methaemoglobinemia}

In methaemoglobinemia, there will be an increase in the concentration of MetHb in the blood. Specific concern for MetHb formation is given for aromatic nitro and amino compounds.

MetHb is formed when the ferrous iron in haemoglobin $(\mathrm{Hb})$ is oxidised to ferric $\left(\mathrm{Fe}^{3+}\right)$ iron. This can occur by oxidation of $\mathrm{Hb}$ within erythrocytes or by the rapid oxidation of free $\mathrm{Hb}$ released from lysed erythrocytes. There are reducing systems available in the erythrocyte to reduce the ferric iron back to the ferrous form, mainly mediated by NADH-diaphorase (synonymous to MetHb reductase). MetHb occurs in healthy individuals at very low concentrations $\leqslant 1 \%$ (human), $0-$ $4 \%$ (rat), $0-2 \%$ (mouse) of the total haemoglobin (Blom, 2001). There is reasonably strong evidence to indicate that there are quantitative differences in the relative sensitivity to MetHb formation between species. In general, the rat, mouse, rabbit, guinea pig and monkey seem significantly less sensitive to the formation of MetHb than humans and dogs. The cat is known to be particularly sensitive to the formation of MetHb and thus may over-estimate the hazard to humans (Smith, 1986, Blom, 2001 and Rockwood et al., 2003).

Haemolytic anaemia may be associated with methaemoglobinemia. MetHb binds oxygen more strongly than $\mathrm{Hb}$ and therefore does not effectively deliver oxygen to tissues. The impact on health of methaemoglobinemia depends on the extent of formation of MetHb (as a proportion of total $\mathrm{Hb}$ ) and can be life threatening when the level exceeds around 50\%. Chronic methaemoglobinemia at low levels (below $10 \%$ ) are generally asymptomatic. However, a blue/gray appearance of the extremities (nails, nose, fingertips and skin) may already occur at slightly raised MetHb (>6\%) in animals and humans. Clinical symptoms of hypoxia due to MetHb includes cyanosis, dyspnoea, fatigue, headache, weakness, dizziness, tachycardia and chocolate brown blood, which appear when the concentration reaches 15-40\% of MetHb (Blom, 2001 and Beutler, 1995).

Populations with a higher risk for developing haemolytic anaemia might be those with hereditary methaemoglobinemia due to NADH-diaphorase deficiency (enzyme catalyzes MetHb reduction) and infants as their NADH diaphorase activity is low.

\subsection{Compensatory mechanisms}

Several effects are seen in animals to compensate the reduction in oxygen carrying capacity caused by the induction of anaemia, haemolytic anaemia, methaemoglobinemia and other changes in erythrocytes. Some of the compensatory effects such as a decrease in the Hb oxygen affinity, increased plasma volume, increased cardiac output, reduction of MetHb to $\mathrm{Hb}$ and shunting away of blood from normally well perfused organs to vital organs are rapid, mainly circulatory responses, which can be seen within a short period after the reduction of the oxygen carrying capacity. In practice, shunting away of blood can be manifested as clinical observations such as pallor of the extremities and mucosae and increased cardiac output can be seen as tachycardia. Shunting away of blood is a clear functional disturbance because the reduced amount of blood reduces the function of the organ. However, when this is only transient due to the delayed onset of compensating mechanisms, this alone is not an indication of serious damage. 
The reduced oxygen supply stimulates peritubular cells in the renal cortex to produce increased amounts of erythropoietin (Epo). Epo stimulates erythropoiesis in the bone marrow, resulting in a release of reticulocytes to the blood stream where they will complete their maturation. Increased reticulocyte counts are probably seen within hours. With increasing demand on the regenerative activity of the bone marrow, extramedullary erythropoiesis can be seen mainly in the spleen and the liver. These compensatory effects can only be seen after days and it may take weeks for the full effect to become evident.

In principle the compensatory changes are adaptive and reversible on cessation of exposure. As long as compensatory responses are expressed as subclinical (non-significant) effects, which do not cause an organ dysfunction by itself and are fully reversible, they are real adaptations. If toxicity exceeds the capacity to compensate the toxic effects (haemolysis), clinical anaemia can be observed. At this stage it must be considered that the normal (physiological) function of the haematopoietic system is disturbed.

\subsection{Toxicological relevance of haemolytic anaemia}

Haemolysis is a toxicological significant adverse effect in itself (cell destruction) and is associated with important secondary effects, which are well known to man. These secondary effects include tissue hypoxia and heart strain (from anaemia), kidney and tissue damage (from free haemoglobin in the circulating blood), decreased tissue viability and oxidative stress (from haemosiderosis), tendency for gall stone formation (from increased production of bilirubin) and the possibility of temporary failure of erythropoiesis (from stress on the bone marrow). These secondary effects may not be observed in repeated dose toxicity studies in animals but should be taken into account when the adversity of haemolysis is considered.

\section{Toxicological indications of haemolytic anaemia}

For most substances, the effect assessment of this hazard and the subsequent need for classification with R48 has to be decided on data from the oral 28 day repeated dose study in rodents. It should be noted that the standard requirements for 28 day studies do not include a number of testing parameters that may give valuable information on the haemolytic anaemia, e.g. there is lack of urinalysis, unconjugated or even total bilirubin, or red cell morphology. If there are data from longer studies (e.g. 90 days or more) of sufficient quality, then these are considered to be more reliable.

The following sections shall outline a range of potential toxicological indicators of haemolytic anaemia and relevant experimental parameters (standard and nonstandard), together with guidance on the interpretation of their toxicological significance for this endpoint. It should be noted that none of these indicators should be evaluated alone but should be considered in context with all of the toxicological effects induced by the substance.

\subsection{Clinical observations}

Clinical observations provide important information in relation to anaemia. Although many of the clinical symptoms of anaemia in man need self-reporting, there are corresponding clinical signs in test animals indicative of reduced or insufficient oxygenation of tissues (hypoxia), such as dyspnoea, cyanosis, pallor and fatigue, the severity of which are likely to be in proportion to the relative extent of the anaemia. Occasionally, jaundice may be a clinical sign of haemolytic 
anaemia. The presence of these signs undoubtedly constitutes significant toxicity and an adverse effect on health.

\subsection{Haematology}

\subsubsection{Haemoglobin concentration, red blood cell count and haematocrit}

Indicators of anaemia are reduced haemoglobin concentration $(\mathrm{Hb})$, red blood cell count (RBC) and haematocrit (Hct). Animals are comparable to humans in that reductions in $\mathrm{Hb}, \mathrm{RBC}$ and $\mathrm{Hct}$ are indicative of anaemia.

In humans the $\mathrm{Hb}$ concentrations in Table 1 can be used to differentiate between the normal state and several levels of anaemia (Harrison's, 2001, SCP, 2002 and World Health Organization, 1968).

Table 1.

Differentiation of human anaemia levels

\begin{tabular}{|l|l|l|}
\hline State & Female & Male \\
\hline Normal & $120-160 \mathrm{~g} \mathrm{Hb} / \mathrm{L}$ & $130-180 \mathrm{~g} \mathrm{Hb} / \mathrm{L}$ \\
\hline Anaemia & $<120 \mathrm{~g} / \mathrm{L}$ & $<130 \mathrm{~g} / \mathrm{L}$ \\
\hline Moderate anaemia & $<100 \mathrm{~g} \mathrm{Hb} / \mathrm{L} \mathrm{F}>-17 \%, \mathrm{M}>-23 \%$ \\
\hline Severe anaemia associated with signs and symptoms & $<70-80 \mathrm{~g} \mathrm{Hb} / \mathrm{L} \mathrm{F}>-38 \%, \mathrm{M}>-38 \%$ \\
\hline Reductions resulting in shunting away blood to vital organs & Reduction of more than $20-30 \mathrm{~g} \mathrm{Hb} / \mathrm{L}$ \\
\hline
\end{tabular}

The human body can compensate for a reduction of up to around $20 \% \mathrm{Hb}$ (20$30 \mathrm{~g} \mathrm{Hb} / \mathrm{l}$ below normal levels of 130-180 and 120-160 g Hb/l blood for males and females, respectively) without any significant clinical effect. In animals, a reduction in $\mathrm{Hb}$ concentration of below $10 \%$ is generally asymptomatic. A reduction of $10 \%$ and above is usually associated with additional biological changes, such as extramedullary haematopoiesis, but is not always associated with any adverse clinical changes indicating hypoxia. It should be noted that the $\mathrm{Hb}$ concentration does not take into account the level of MetHb.

Reductions in Hct and RBC counts should also be considered as indicators of anaemia. However, these indicators are considered to be less determinative for anaemia than the $\mathrm{Hb}$ concentration because the Hct also depends on the amount of other blood cells and the RBC counts do not accurately determine the oxygen transport capacity as changes in the amount of haemoglobin per erythrocyte are not reflected.

Compared to the 100-120 days of erythrocyte life span in humans, the red cell life span in rats is only 50 days. Since haemolytic anaemia is characterised by the reduced life span of erythrocytes, data from 28-day repeated dose studies are insufficient to cover the full cell cycle of erythrocytes. Similar to age-related changes in humans, the erythrocyte counts and $\mathrm{Hb}$ concentration decreases in rats after 16-20 months of age (Bailly and Duprat, 1990).

In conclusion, a reduction in $\mathrm{Hb}, \mathrm{RBC}$ and $\mathrm{Hct}$ is an adverse but reversible effect. Therefore, this effect should be used for classification if it is severe or when there 
is clear evidence for marked organ dysfunction. This is the case if there is a reduction in $\mathrm{Hb}$ at or above $20 \%$ (also as a stand-alone criteria) or if $\mathrm{Hb}$ reduction is at or above $10 \%$ and indicators of dysfunctions or organ damage, as given in the examples in Appendix on application of the classification criteria for R48, are present.

\subsubsection{Reticulocytes}

Precursors of erythrocytes are produced in the bone marrow and released in the blood as reticulocytes. The reticulocytes ripen for one or two days in the blood or in the spleen to erythrocytes. Normally, the reticulocyte counts in humans range from 1 to $2 \%$ and reflect the daily replacement of erythrocytes in the circulation. A reduction of the oxygen transporting capacity of the blood will result in an increased production of precursors in the bone marrow and an increased reticulocyte count in the blood. An increase in reticulocytes is correlated with an increase in MCV. If there is substantial anaemia, the red cell number will be reduced, and the relative proportion of reticulocytes will be abnormally high. Consequently it's important to take into account the absolute count of reticulocytes and not the \% (Absolute count of reticulocytes $=\%$ reticulocytes $\times$ erythrocytes number/100). This effect is adaptive and reversible. In the absence of other indicators of haemolytic anaemia, it is not considered to be adverse.

\subsubsection{Additional erythrocyte parameters}

The following secondary parameters of erythrocytes are often provided in repeated dose study reports:

Haematocrit (HCt) or Packed cell volume (PCV: \%): often reduced in haemolytic anaemia

Mean Corpuscular Haemoglobin Concentration (MCHC: $g / d l)$ : amount of haemoglobin per unit erythrocyte volume: often reduced in haemolytic anaemia or increased in case of massive intravascular haemolysis $(\mathrm{Hb} \times 100 / \mathrm{PCV})$.

Mean Corpuscular Volume ( $M C V: f L)=$ average volume of the erythrocyte: often increased in haemolytic anaemia as the result of reticulocytosis (PCV $\times 10 / R B C)$.

Mean corpuscular haemoglobin ( $\mathrm{MCH}: \mathrm{pg}$ ): average amount of haemoglobin per cell: often increased in haemolytic anaemia $(\mathrm{Hb} \times 10 / \mathrm{RBC})$.

Red cell volume distribution width (RDW): variation of the size of the erythrocytes (anisocytosis): often increased in haemolytic anaemia.

In cases of chronic haemolytic anaemia erythrocytes are generally larger (MCV increased) and contain more haemoglobin ( $\mathrm{MCH}$ increases) but the amount of haemoglobin per volume unit of erythrocytes is reduced (MCHC decreases). These changes are adaptive and reversible.

Red cell survival: the survival time of red blood cells in animals and humans can be determined using chromium studies. By definition this parameter is reduced in haemolytic anaemia. 


\subsubsection{Methaemoglobinemia}

Methaemoglobin is usually not studied in detail in standard animal studies. Therefore, there is limited evidence to allow a correlation to be made between MetHb concentration and induction of clinical symptoms in animals. Mammalian species differences (the rat is less sensitive than the man) with respect to MetHb reductase activity and onset and duration of MetHb is known and should be taken into consideration (Smith, 1986, Rockwood et al., 2003 and Blom, 2001).

It should also be noted that many older studies are inadequate with respect to the measured level of MetHb in particular the time point at which blood was taken and subsequently measured (Blom, 2001). MetHb is usually rapidly reduced back to $\mathrm{Hb}$ and so measured levels may potentially underestimate the true extent of oxidation that has occurred. As the kinetics of MetHb production is mostly unknown and MetHb levels may return to normal values within $24 \mathrm{~h}$, the blood sampling is often not conducted at peak levels. Also, the time point of the performance of the measurements is critical, as MetHb levels in blood samples tend to increase by auto-oxidation.

An indication of increased formation of MetHb is often, but not always, the presence of Heinz bodies in the red blood cells. These are essentially precipitates of damaged $\mathrm{Hb}$ in erythrocytes that can cause a loss of membrane integrity, and reduce cell deformability (important for proper circulation in capillaries) leading to lysis or removal. Erythrocytes damaged by methaemoglobin formation can lyse in the bloodstream or can be removed from the bloodstream by the spleen and other organs. Heinz bodies are more persistent than MetHb and so their presence may be a more robust indicator of MetHb formation than measurements of blood MetHb concentration.

\subsubsection{Abnormal red blood cell morphology}

The morphology of the red blood cells can be correlated with the severity of the haemolytic process. Apart from reticulocytes and Heinz bodies as indicators of anaemia and methaemoglobinemia, other morphological changes in red blood cells can be indicative of haemolytic anaemia:

Howell-Jolly bodies (micronuclei) are spherical eccentrically placed inclusions in reticulocytes (rarely in erythrocytes), they represent nuclear (chromosomal) remnants indicating abnormal mitosis and have been observed in relation to haemolytic anaemia. They have a reduced amount of cell membrane and a hyperchromatic colour.

Cabot rings are inclusions with the shape of thread-like rings and convolutions in erythrocytes. They are suggested to be of nuclear derivation as they can be stained with stains specific for chromatin. Cabot rings have been observed in the same haemolytic disorders as Howell-jolly bodies but their source is less clear.

Spherocytes are spheroid rather than biconcave disk-shaped erythrocytes. They have relatively small corpuscles, which stain bright red. They are characteristic for hereditary spherocytosis and may be found in other forms of haemolytic anaemia. The corpuscular haemoglobin concentration of spherocytes is greater than normal and this is reflected by an increase in $\mathrm{MCHC}$.

Schistocytes are fragments of red corpuscles, which usually take the form of tiny elliptical, triangular or otherwise irregular shapes. They are seldom in normal blood and they are presumably products of corpuscular fragmentation, which 
usually are sieved out of the circulation by the spleen. Their presence is good evidence of haemolytic anaemia.

Irregular contracted red corpuscles, shrunken and distorted forms have been observed following ingestion of haemolytic poisons.

Triangular cells which have rarely more than two spines are microcytic and stain deeply. They have been observed in association with uremia and haemolytic anaemia and are thought to be prehaemolytic forms.

Poikilocytes are erythrocytes with an abnormal shape and are commonly observed in immune-mediated haemolytic anaemia. They are an indication of reduction of the flexibility and functioning of the erythrocytes.

Target or Mexican hat cells are hypochromatic red cells where the corpuscles have a central rounded area of pigmented material surrounded by a clear ring without pigment, outside of which is the pigmented border of the corpuscles, making the corpuscles look like a hat. These cells can be seen in haemolytic disorders.

Echinocytes are morphologically altered red blood cells that appear to have numerous, fine, uniform spicules throughout the cell membrane. Several disease processes and toxins have been found to alter the red blood cell membrane, leading to the formation of echinocytes.

The presence of abnormal erythrocyte morphology can be indicative of haemolytic anaemia and should be considered a toxicologically adverse effect, as they may be associated with other haematotoxic effects.

\subsection{Blood chemistry}

\subsubsection{Bilirubin}

Increased levels of bilirubin although not specific may be indicative of an increased degradation of heme. Therefore, in the case of anaemia, hyperbilirubinemia (mainly unconjugated) is highly suggestive of increased haemolysis of erythrocytes, although its absence does not exclude the diagnosis. The bilirubin levels will decrease when the haemolysis is ended. Although high bilirubin levels have not always been associated with jaundice in rodents, their presence is considered to be relevant to both animals and man in the context of haemolytic anaemia.

\subsubsection{Lactate dehydrogenase}

Lactate dehydrogenase (LDH) is released from the erythrocytes during haemolysis. Increased LDH activity may thus be an indication of haemolysis. However, it should be noted that this enzyme is not specific for haemolysis.

\subsubsection{Erythropoietin}

In response to tissue hypoxia due to anaemia, the production of erythropoietin (Epo) is increased in the kidney. This results in stimulation of erythropoiesis in the bone marrow and should be regarded as induction of a compensatory mechanism in response to anaemia. 


\subsubsection{Free haemoglobin in the circulating blood}

During intravascular haemolysis, free haemoglobin can be present in the blood in the reduced or oxidised form ( $\mathrm{Hb}$ and MetHb respectively) and elevations may cause adverse effects. If the total amount of free haemoglobin in the circulating blood is greater than the quantity capable of binding with haptoglobin or other binding proteins much of the excess will leak through the glomerular membranes into the kidney tubules. If this amount is still slight, it can be reabsorbed through the tubular epithelium and will probably cause no harm; if it is great, then only a small percentage is reabsorbed. Water continues to be reabsorbed, causing the tubular haemoglobin concentration to rise so high that it precipitates and blocks the tubules. Therefore elevations in free $\mathrm{Hb}$ in the blood should be taken into account in the evaluation of the adversity of haemolytic anaemia.

\subsubsection{Parameters for evaluating iron status}

There are some useful screening parameters for iron homeostasis in serum:

Serum iron: elevated in systemic iron overload (can also be high in marrow suppression)

Serum ferritin: as an indicator of the body iron store (Watanabe et al., 2001), high values of ferritin reflect high iron loading, but hyperferritinemia may also occur in other (inflammatory/congenital) diseases without iron overload.

Calculation of serum transferrin saturation: percentage increases in iron overload.

Total iron-binding capacity (TIBC): is inversely related with body iron stores.

\subsection{Urinalysis}

\subsubsection{Haemoglobinuria}

The presence of haemoglobin in the urine leading to 'red urine' is indicative of severe intravascular haemolysis. However, red urine can have other causes. At normal perfusion pressures, the plasma haemoglobin concentrations in humans must exceed $30 \mathrm{mg} / \mathrm{dl}$ before haemoglobinuria ensues. In animals this effect is often seen in combination with clinical effects, including mortality.

\subsubsection{Haemosiderinuria}

Haemosiderinuria is indicative of tubular cell death following intravascular haemolysis because amounts of free haemoglobin in the blood reach the kidney where the haemoglobin is degraded after resorption from urine. The iron is stored as haemosiderin in the tubular cells. Subsequently tubular cells with haemosiderin are sloughed into the urine. This effect is seen after a delay of a few days after the start of the haemolysis and may persist for several days after haemolysis has terminated. Accordingly the detection of haemosiderin in the urine is indicative of either ongoing or recent haemolysis. Haemosiderinuria may be indicative of serious damage in the kidney but this should be supported by histopathological findings.

When the renal tubular cells are sloughed into the urine, iron can be detected as intra- and extracellular "Prussian Blue deposits" in the urinary sediment. 


\subsection{Bone marrow}

Increased erythropoiesis in the bone marrow (evidenced by hyperplasia) can be an adaptive response, which should be sufficient to counter some degree of subclinical anaemia without adverse effect, and be reversible on cessation of exposure. Often the increased erythropoiesis in the bone marrow caused by haemolytic anaemia also stimulates the leukopoietic tissue to produce a high number of leucocytes and platelets at the same time. With an increasing demand for new erythrocytes, an increase in reticulocytes in the blood and extramedullary erythropoiesis in the liver and spleen and possibly other tissues may be observed. These findings may represent changes within a normal range of response to anaemia, or may indicate that the ability of the body to cope with the level of anaemia experienced is being challenged.

It should be noted that interstrain differences in the participation of different bone regions may exist in mice (Vacha et al., 1982). Data on the intensity of medullary erythropoiesis could only be compared between studies when the same strain was used and the same localisation was examined.

During prolonged haemolysis, an increase in haemosiderin accumulation is expected to be seen in the bone marrow. The bone structure may also be affected in highly regenerative (haemolytic) anaemia. In humans, prolonged erythroblastic proliferative activity causes changes in the bone structure and induces periosteal thickening of skull and facial bones, spine and ribs (Tunaci et al., 1999 and Schaefer et al., 1989). In dogs with inherited pyruvate kinase deficiency of erythrocytes causing chronic haemolytic anaemia, osteosclerosis/myelofibrosis of large bones was associated with marked erythroid hyperplasia (Giger and Noble, 1991).

\subsection{Spleen}

Macroscopic effects such as increased spleen weight, enlarged spleen, splenomegaly and dark spleen with irregular rough appearance could be indicative of increased degradation of erythrocytes including haemosiderin accumulation, extramedullary haematopoiesis or both. Splenomegaly and related findings accompanying haemolytic anaemia can be seen in test species and in humans.

In spleen homogenate, products of lipid peroxidation (e.g. malondialdehyde and 4-hydroxy-2(E)-nonenal), iron content/g, total iron content/spleen and (cheletable) free iron content/g might be useful parameters to characterise iron accumulation and oxidative damage (Ciccoli et al., 1999).

At the microscopic level a number of effects may occur in response to haemolytic anaemia such as increased red cell phagocytosis (erythroclasia), increased extramedullary haematopoiesis, increased accumulation of haemosiderin, increased red pulp and capsular fibrosis and perisplenocapsulitis. Other related findings are congestion and dilatation and accumulation of macrophages in the sinus and may be prominent in the early phase of haemolytic anaemia. In chronic haemolysis, fibrosis can be intermingled with areas of fatty metamorphosis. These findings may alter the relationship between the red and white pulp leading to a strong enlargement of the red pulp. To demonstrate the increase in fibrotic tissue special stainings for collagen fibres may be necessary.

Since a low extent of haemosiderosis is a normal age-related lesion that may show some degree of interindividual variation, only clear increases in 
haemosiderin deposition compared to the internal control group should be considered as treatment-related effects. Small increases of haemosiderosis may often be overlooked because of other changes in the spleen.

Haemosiderin appears in standard haematoxylin and eosin stain (H\&E) paraffin sections as clumps of intracellular gold-yellow pigment granules and is identified by Perl's Prussion blue staining. In cases without accurate staining for haemosiderin, an increase in intracellular granular pigments can be used as a surrogate for haemosiderin accumulation.

It should also be noted that the most prominent histopathological finding in chronic haemolysis are haemosiderin deposition and increased erythropoiesis. In a standard H\&E paraffin section multiple layers of cells with pigment granules often make it difficult to detect other simultaneous findings unless they are severe graded or special stains or other methods are applied. Excessive erythrophagocytosis may also generate conglomerates of lipofuscin (pigment) deposition reflecting oxidative breakdown of lipids as remnants of degenerated lysosomes and crystalloid residuals from red cell destruction (Danse and Crichton, 1990).

The decision whether these changes indicate serious damage should be based on the microscopic evaluation of the spleen.

\subsection{Liver}

Macroscopic effects such as increased liver weight, enlarged liver or darkened liver could be indicative of extramedullary haematopoiesis or uptake of residues from haemolysed erythrocytes (haemosiderosis). Firm or irregular shaped liver lobules may indicate advanced lesions.

Liver homogenates can be used to characterise the iron loading by increases in the iron content in the dry mass, the ferritin content, the total non-heme iron (Watanabe et al., 2001) and so-called free iron (Khan et al., 2002). In individuals exposed chronically to haemolytic substances, non-invasive techniques such as computed tomography and magnetic resonance imaging might be considered for screening on iron accumulation. If available, the iron content could also be quantified in biopsy samples (Beilby et al., 1999).

At the microscopic level a number of effects may occur in response to haemolytic anaemia such as increased red cell phagocytosis, Kupffer cell activation, increased accumulation of haemosiderin in endothelial (Kupffer) cells and sometimes in hepatocytes (and bile duct epithelium), increased extramedullary haematopoiesis, increased fibrosis, and cirrhosis. Hepatocellular necrosis/single cell necrosis, inflammation and hepatocytomegaly are also observed in studies on haemolytic substances. The interpretation as to whether these changes are related to haemolysis or idiopathic hepatotoxicity may be difficult.

Haemosiderin deposition in the liver is a pathological condition. A prominent accumulation of haemosiderin pigment in sinusoidal Kupffer cells and hepatocytes gives an indication of intravascular haemolysis. More subtle lesions such as increases in collagen fibre production as early indicators of fibrosis, activation of Kupffer cells, transformation of sinusoidal stellate cells or single cell degeneration may be associated with liver haemosiderosis. A more accurate method than the standard H\&E section is required to show their presence (see Section 3.6). 
The decision whether these changes indicate serious damage should be based on the microscopic evaluation of the liver.

\subsection{Kidney}

Macroscopic effects such as discoloration or blackening of the cortex region of the kidney could also be attributed to erythrocyte destruction.

At the microscopic level a number of effects may occur in response to haemolytic anaemia such as eosinophilic inclusions in cortical tubular epithelium, haemosiderin accumulation in tubular cells and sometimes in the glomeruli and interstitium, sloughing of haemosiderin-laden cells, tubular cell necrosis, atrophy and regeneration, and in severe cases interstitial fibrosis. Special stains are recommended to allow discrimination from other intracellular inclusions or to detect glomerulosclerosis Section 3.6.

The decision whether these changes indicate serious damage should be based on the microscopic evaluation of the kidney and should also be correlated to alterations in urine and blood chemistry parameters.

\subsection{Effects in multiple organs}

\subsubsection{Extramedullary haematopoiesis}

Extramedullary haematopoiesis is a normal feature in young animals. It is less obvious in healthy adults, but will readily increase in animals at any age in response to haematopoietic or other (neoplastic) insults. Extramedullary haematopoiesis occurs mainly in the spleen but also in the liver, lymph nodes and perinephric tissues. With increasing demand on the regenerative activity of the bone marrow, extramedullary erythropoiesis can be seen. In untreated adult rodents a significant fraction of the total body haematopoietic activity occurs in the spleen ( $<5 \%$ in rats, more pronounced in mice) and the spleen was reported as being the first site to respond on increased demand (cited from Seifert and Marks, 1985). The degree of increased activity in the spleen should be estimated in addition to the haematopoietic activity at other organs with haemopoietic potential.

This effect is adaptive and may be reversible. This may indicate that the ability of the body to cope with the level of anaemia experienced is being challenged.

\subsubsection{Haemosiderosis}

Haemosiderosis is the deposition of haemosiderin-bound iron in several organs, usually the liver, spleen, kidney and bone marrow. The medullary cords and subcapsular sinuses of associated lymph nodes can also be affected and may appear expanded by macrophages with dense dark brown (haemosiderin) pigment. Its occurrence in multiple organs reflects the major type of haemolysis (intra- vs. extravascular). On the other hand multiplicity of organs affected reflects the severity of haemolysis, e.g. in an intravascular haemolysis of minor severity grade haemosiderinuria (and its secondary kidney damage) should not be expected as long as the protein binding capacity for haemoglobin is sufficient. When the extent of haemolysis is extensive haemosiderin may be deposited in the liver, spleen, kidney, bone marrow and other organs. 
Haemosiderosis is defined as a marked increase in haemosiderin accumulation in organs compared to the normal status, and thereby is a hallmark of secondary iron overload. Since it is not fully reversible and is associated with several adverse effects in the organs including fibrosis, and cell death, marked haemosiderosis in multiple organs is clearly an adverse effect. Besides the specific cytopathological effects of iron (haemosiderin) overload at tissue sites in one or multiple organs, that could be examined by morphological, biochemical, and analytical parameters suggested above, a systemic imbalance of iron homeostasis may also develop in chronic haemolysis (see Section 3.3.5).

\section{Acute vs. repeated dose toxicity}

Haemolytic anaemia can be induced by a single dose of a substance and by repeated dosing. Acute and chronic toxicity are separate endpoints that are examined by separate testing and may result in different classification proposals. Occasionally, results from repeated dose studies are considered for assessment of acute toxicity.

In general, a substance is classified for acute toxicity when the haemolytic anaemia results in marked toxicity or deaths at the appropriate dose level in an acute test that are clearly above those dose levels in repeated dose studies. In some cases, haemolysis-related sudden deaths are seen during the first days of exposure in a repeated-dose test at markedly lower doses than the range estimated in acute toxicity testing. If mortalities occur only during the first days (2-3 days), they should be evaluated for acute toxicity.

A haemolytic substance may be classified as very toxic, toxic or harmful due to its acute toxicity (e.g. by mortalities) in addition to a classification as toxic or harmful with R48 for its chronic haemolytic effects. For the majority of substances the cause of death being of haemolytic or another nature is not identified in acute toxicity studies. Even, if severe acute haemolysis was identified as a cause of death or of severe toxic signs in acute toxicity testing, the same substance could be classified for chronic toxicity depending on the nature and severity of effects due to chronic haemolytic anaemia seen at the appropriate dose levels.

Other non-lethal effects with limited occurrence during the first days of treatment may be attributed to true acute effects or to transient effects within the cascade of secondary responses to persistent haemolysis. Such transient effects may be explained by the delayed onset of compensatory responses to continuous haemolysis, e.g. cyanosis occurring during the first days of treatment when compensatory haematopoiesis needs several days for maximum activation. Therefore, transient effects (reversible within the exposure period) are not regarded as serious damage.

\section{Conclusion}

Substance-induced chronic haemolytic anaemia is considered to be an adverse effect that can cause serious damage to health and thus can warrant classification with R48 within the EU classification system. It is evident that anaemia describes a continuum of effects, from sub-clinical to potentially lethal in severity. Overall, the interpretation of study findings requires an assessment of the totality of findings, to judge whether they constitute an adaptive response or an adverse toxicologically significant effect. Overt clinical signs of hypoxia represent a clear undesirable impact on health. As described above, other changes can be seen to lie on a spectrum of responses. The main function of the erythrocytes is the transport of oxygen to the tissues. A reduction of the transport capacity is 
therefore a functional disturbance. However, this reduction can be compensated by several mechanisms. In general, compensating or adaptive responses in itself do not warrant classification but can be used to assess the increased destruction of erythrocytes and accumulation of their residues. Therefore, adaptive parameters can be used as guidance for classification if the serious damage was not investigated or not seen due to species differences.

The determination of toxicological significance of the effects, in the context of classification, must take into account the nature and time-course of the clinical and pathological effects observed, the severity of the effects or degree of change in key parameters, and the dose-response relationship of the effects etc. to judge the significance of the impact on health. Guidance values on what magnitude of change in key parameters (e.g. Hb and MetHb level) are considered to be biologically significant are useful, but the emphasis should be on interpretation of the whole biological picture to judge the impact on health. Expert judgement is required to ascertain whether the pattern of changes observed indicates that the adaptive capacity has been exceeded, causing organ dysfunction, and therefore indicates adverse toxicity. It is anticipated that many of these observations, e.g. erythropoiesis, would be reversible on cessation of exposure, but this does not necessarily detract from them being considered to be a toxic response occurring during exposure.

The harmonised position of the working group on haemolytic anaemia on the extended classification criteria is available in Appendix A. The criteria were accepted by the EU Commission Working Group on the Classification and Labelling of Dangerous Substances in September 2004.

\section{References}

Arrezzini et al., 2003 B. Arrezzini, B. Lunghi, G. Lungarella and C. Gardi, Iron overload enhances the development of experimental liver cirrhosis in mice, Int. J. Biochem. Cell Biol. 35 (2003), pp. 486495.

Aydogdu et al., 2001 I. Aydogdu, R. Sari, R. Ulu and A. Sevinc, The frequency of gallbladder stones in patients with pernicious anaemia, J. Surg. Res. 10 (2001) (2), pp. 120-123.

Azemoto et al., 1996 R. Azemoto, Y. Tsuchiya, T. Ai, H. Murayama, Y. Nakagawa, H. Saisho and M. Ohto, Does gallstone formation after open cardiac surgery result only from latent haemolysis by replaced valves?, Am. J. Gastroenterol. 9 (1996) (10), pp. 2185-2189.

Bader-Meunier et al., 2001 B. Bader-Meunier, F. Gauthier, F. Archambaud, T. Cynober, F. Mielot, J.-P. Dommergues, J. Warszawski, N. Mohandas and G. Tchernia, Long-term evaluation of the beneficial effect of subtotal splenectomy for management of hereditary spherocytosis, Blood 9 (2001) (2), pp. 399-403.

Bailly and Duprat, 1990 Y. Bailly and P. Duprat, Normal blood cell values, rat. In: T.C. Jones, J.M. Ward, U. Mohr and R.D. Hunt, Editors, Hemopoietic System, Springer-Verlag, Berlin (1990), pp. 2738.

Baker and DeMaeyer, 1979 S.J. Baker and E.M. DeMaeyer, Nutritional anemia: its understanding and control with special reference to the work of the World Health Organization, Am. J. Clin. Nutr. 3 (1979) (2), pp. 368-417.

Barr et al., 1998 F. Barr, L. Brabin, S. Agbaje, F. Buseri, J. Ikimalo and N. Briggs, Reducing iron deficiency anaemia due to heavy menstrual blood loss in Nigerian rural adolescent, Public Health Nutr. 1 (1998) (4), pp. 249-257.

Beilby et al., 1999 J.P. Beilby, A.W. Prins and N.T. Swanson, Determination of hepatic iron concentration in fresh and paraffin-embedded tissue, Clin. Chem. 45 (1999), pp. 573-574. 
Beutler, 1995 E. Beutler, Methaemoglobinemia and other causes of cyanosis. In: E. Beutler, M.A. Lichtman, B.S. Coller and T.J. Kipps, Editors, Williams Hematology (fifth ed.), McGraw-Hill, Inc., New York (1995), pp. 654-662.

Blom, 2001 Blom, M. in RIVM Rapport 601516007 Luttik R; Raaij MTM van (eds) 2001 Factsheets for the (eco)Toxicological Risk Assessment Strategy of the National Institute of Public Health and the Environment (RIVM).

Bonatsos et al., 2001 G. Bonatsos, K. Birbas, K. Toutouzas and N. Durakis, Laparoscopic cholecystectomy in adults with sickle cell disease, Surg. Endosc. 1 (2001) (8), pp. 816-819.

Ciccoli et al., 1999 L. Ciccoli, M. Ferrali, V. Rossi, C. Signorini, C. Alessandrini and M. Comporti, Hemolytic drugs aniline and daponse induce iron release in erythrocytes and increase the free iron pool in spleen and liver, Toxicol. Lett. 110 (1999), pp. 57-66.

Danse and Crichton, 1990 L.H.J.C. Danse and D.N. Crichton, Pigment deposition, rat, mouse. In: T.C. Jones, J.M. Ward, U. Mohr and R.D. Hunt, Editors, Monographs on Pathology of Laboratory Animals Sponsored by the International Life Sciences Institute. Hemopoietic System, Springer-Verlag, Berlin (1990), pp. 226-232.

Diagne et al., 1999 I. Diagne, M. Badiane, C. Moreira, H. Signate-Sy, O. Ndiaye, P. Lopez-Sall, G. Preira-Sylla, B. Camara, S. Diouf, A. Diack-Mbaye and M. Fall, Lithiase biliaire et drepanocytose homozygote en pediatrie a Dakar (Senegal).[Cholelithiasis and homozygote drepanocytosis in children in Dakar (Senegal)], Arch. Pediatr. 6 (1999) (12), pp. 1286-1292.

Duellmann et al., 1992 J. Duellmann, U. Wulfhekel, P. Nielsen and H.C. Heinrich, Iron overload of the liver by trimethylhexanoylferrocene in rats, Acta Anat. 143 (1992), pp. 96-108.

Emerit et al., 2001 J. Emerit, C. Beaumont and F. Trivin, Iron metabolism, free radicals, and oxidative injury, Biomed. Pharmacother. 55 (2001), pp. 333-339.

Erslev and Beutler, 1995 H.M. Erslev and V. Beutler, Structure and function of haemoglobin. In: E. Beutler, M.A. Lichtman, B.S. Coller and T.J. Kipps, Editors, Hematology (fifth ed.), McGraw-Hill, Inc., New York (1995), pp. P417-P438.

EU, 1967 EU, 1967. Council Directive 67/548/EEC of 27 June 1967 on the Approximation of Laws, Regulations and Administrative Provisions Relating to the Classification, Packaging and Labelling of Dangerous Substances. O.J. No. P196,16. 8. 1967, p. 1.

Fairbanks and Baldus, 1995 V.F. Fairbanks and W.P. Baldus, Iron overload. In: E. Beutler, M.A. Lichtman, B.S. Coller and T.J. Kipps, Editors, Hematology (fifth ed.), McGraw-Hill, Inc., New York (1995), pp. P529-P535.

Giger and Noble, 1991 U. Giger and N.A. Noble, Determination of erythrocyte pyruvate kinase deficiency in Basenjis with chronic hemolytic anemia, JAVMA 198 (1991), pp. 1755-1761.

Goswami et al., 2001 R.P. Goswami, D. Banerjee and D. Shah, Cholelithiasis in a child-an unusual presentation of Wilson's disease, J. Assoc. Physicians India 49 (2001), pp. 1118-1119.

Harada et al., 1998 Y. Harada, M. Iwai, M. Kakusui, T. Mori, K. Tada, Y. Ishii, T. Okanoue and K. Kashima, Activated hepatic stellate cells participate in liver fibrosis in a patient with transfusional iron overload, J. Gastroenterol. 33 (1998), pp. 751-754.

Harrison's, 2001 Harrison's Principles of Internal Medicine 2001. Braunwald, E., Hansen, S.L., Fauci, A.S., Longo, D.L., Kasper, D.L., Jameson, J.L. (eds.), McGraw-Hill, New York.

Hertz et al., 1992 T. Hertz, J. Dullmann and P. Nielsen, Transport of siderotic Kupffer cells to the lung and bronchial excretion of iron in experimental iron-overload, Acta Anat. 145 (1992), pp. 378-381.

Inan et al., 1998 C. Inan, K. Kiline, E. Kotiloglu, H.O. Akman, I. Kilic and J. Michl, Antioxidant therapy of cobalt and vitamin E in hemosiderosis, J. Lab. Clin. Med. 132 (1998), pp. 157-165.

Izaks et al., 1999 G.J. Izaks, R.G.J. Westendorp and D.L. Knook, The definition of anemia in older persons, JAMA 281 (1999) (18), pp. 1714-1717. 
Khan et al., 2002 M.F. Khan, X. Wu, U.R. Tipnis, G.A.S. Ansari and P.J. Boor, Protein adducts of malondialdehyde and 4-hydroxynonenal in livers of iron loaded rats: quantitation and localization, Toxicology 173 (2002), pp. 193-201.

Leonardi and Ruol, 1960 P. Leonardi and A. Ruol, Renal hemosiderosis in the hemolytic anemias: diagnosis by means of needle biopsy, Blood 16 (1960), p. 1029.

Marchetti et al., 1998 M. Marchetti, S. Quaglini and G. Barosi, Prophylactic splenectomy and cholecystectomy in mild hereditary spherocytosis: analyzing the decision in different clinical scenarios, J. Intern. Med. 244 (1998) (3), pp. 217-226.

Matsuno et al., 1985 T. Matsuno, M. Mori and M. Awai, Distribution of ferritin and hemosiderin in the liver, spleen and bone marrow of normal, phlebotomized and iron overloaded rats, Acta Med. Okayama 39 (1985), pp. 347-360.

Miyazaki et al., 2002 E. Miyazaki, J. Kato, M. Kobune, K. Okumura, K. Sasaki, N. Shintani, P. Arosio and Y. Niitsu, Denatured $\mathrm{H}$-ferritin subunit is a major constituent of haemosiderin in the liver of patients with iron overload, Gut 50 (2002), pp. 413-419.

Nyska et al., 2004 A. Nyska, J.K. Haseman, R. Kohen and R.R. Maronpot, Association of liver hamangiosarcoma and secondary iron overload in B6C3F1 mice-the National Toxicology Program experience, Toxicol. Pathol. 32 (2004), pp. 222-228.

Parkes and Templeton, 2003 J.G. Parkes and D.M. Templeton, Modulation of stellate cell proliferation and gene expression by rat hepatocytes: effect of toxic iron overload, Toxicol. Lett. 144 (2003), pp. 225-233.

Penninx et al., 2003 B.W. Penninx, J.M. Guralnik, G. Onder, L. Ferrucci, R.B. Wallace and M. Pahor, Anemia and decline in physical performance among older persons, Am. J. Med. 115 (2003) (2), pp. 104-110.

Riede et al., 1989 U.-N. Riede, H.-E. Schaefer, H. Denk and M. Stolte, Hepatopankreatisches system. In: U.-N. Riede, H.-E. Schaefer and H. Wehner, Editors, Allgemeine und spezielle Pathologie, Georg Thieme Verlag Stuttgart (1989), pp. 669-723.

Rockwood et al., 2003 G.A. Rockwood, K.R. Armstron and S.I. Baskin, Species comparison of methemoglobin reductase, Exp. Biol. Med. 228 (2003), pp. 79-83.

Sandler et al., 1999 A. Sandler, G. Winkel, K. Kimura and R. Soper, The role of prophylactic cholecystectomy during splenectomy in children with hereditary spherocytosis, J. Pediatr. Surg. 34 (1999) (7), pp. 1077-1078.

Schaefer et al., 1989 H.-E. Schaefer, E.W. Hernst and U.-N. Riede, Hämatopoetisches und lymphatisches system. In: U.-N. Riede, H.-E. Schaefer and H. Wehner, Editors, Allgemeine und spezielle Pathologie, Georg Thieme Verlag Stuttgart (1989), pp. 471-528.

SCP, 2002 SCP (Scientific Committee on Plants), (2002). Opinion on Specific Questions from the Commission Concerning the Evaluation of Indoxacarb in the Context of Council Directive 91/414/eec. <http://europa.eu.int/comm/food/fs/sc/scp/out132_ppp_en.pdf>.

Seifert and Marks, 1985 M.F. Seifert and S.C. Marks, The regulation of hemopoiesis in the spleen, Experientia 41 (1985), pp. 192-199.

Selden et al., 1980 C. Selden, M. Owen, J.M. Hopkins and T.J. Peters, Studies on the concentration and intracellular localization of iron proteins in liver biopsy specimens from patients with iron overload with special reference to their role in lysosomal disruption, Br. J. Haematol. 44 (1980), pp. 593-603.

Smith, 1997 J.E. Smith, Iron metabolism and its disorders. In: J.J. Kaneko, J.W. Harvey and M.L. Bruss, Editors, Clinical Biochemistry of Domestic Animals, Academic Press, New York (1997), pp. 223239.

Smith, 1986 R.P. Smith, Toxic Responses of the Blood, Casarett and Doull's Toxicology (third ed.), Macmillan Publishing Company, New York, Toronto, London (1986). 
Sprague et al., 2003 W.S. Sprague, T.B. Hackett, J.S. Johnson and C.J. Swardson-Olver, Hemochromatosis secondary to repeated blood transfusions in a dog, Vet. Pathol. 40 (2003), pp. 334337.

Tapiero et al., 2001 H. Tapiero, L. Gate and K.D. Tew, Iron: deficiencies and requirements, Biomed. Pharmacother. 55 (2001) (6), pp. 324-332.

Tiniakos and Williams, $1988 \mathrm{G}$. Tiniakos and R. Williams, Cirrhotic process, liver cell carcinoma and extrahepatic malignant tumors in idiopathic haemochromatosis, Appl. Pathol. 6 (1988), pp. 128-138.

Tunaci et al., 1999 M. Tunaci, A. Tunaci, G. Engin, B. Ozkorkmaz, G. Dincol, G. Acunas and B. Acunas, Imaging features of thalassemia, Eur. Radiol. 9 (1999), pp. 1804-1809.

Vacha et al., 1982 J. Vacha, J. Hola, J. Dungel and V. Znojil, The distribution of erythropoiesis over the various anatomical regions of the erythropoietic system in some inbred strains of mice, Exp. Hematol. 10 (1982), pp. 768-773.

Watanabe et al., 2001 K. Watanabe, Y. Yamashita, H. Ohgawara, M. Sekiguchi, N. Satake, K. Orino and S. Yamamoto, Iron content of rat serum ferritin, J. Vet. Med. Sci. 63 (2001), pp. 587-589.

Weinberg, 1983 E.D. Weinberg, Iron in neoplastic disease, Nutr. Cancer. 4 (1983), pp. 223-233.

World Health Organization, 1968 World Health Organization. 1968. Nutritional Anaemias: Report of a WHO Scientific Group. Geneva, Switzerland: World Health Organization.

Zhou et al., 2000 X.J. Zhou, Z. Laszik, X.Q. Wang, F.G. Silva and N.D. Vaziri, Association of renal injury with increased oxygen free radical activity and altered nitric oxide metabolism in chronic experimental hemosiderosis, Lab. Invest. 80 (2000), pp. 1905-1914.

Zuyderhoudt et al., 1983 F.M. Zuyderhoudt, J.W. Sindram, J.J. Marx, G.G. Jorning and J. van Gool, The amount of ferritin and hemosiderin in the livers of patients with iron-loading diseases, Hepatology 3 (1983), pp. 232-235.

\section{Appendix A. Application of the classification criteria for R48}

Classification of haemolytic anaemia follows the generally accepted R48 criteria as laid down in: Annex VI to Directive 67/548/EEC (original text of Annex VI in italics).

Substances should be classified with R48 if serious damage (clear functional disturbance or morphological change that has toxicological significance) is likely to be caused by repeated or prolonged exposure by any appropriate route. It is particularly important when these changes are irreversible. Classification also depends on the dose level, the exposure period and the exposure route. For the oral route classification is warranted when these effects are seen at levels of $150 \mathrm{mg} / \mathrm{kg} \mathrm{bw} /$ day or lower in a 28 day study. Serious effects at $50 \mathrm{mg} / \mathrm{kg}$ bw/day warrant classification in a 90 day study. If results of studies of more than one duration are available, then those from the study of the longest duration should normally be used. According to the guidelines (Annex VI of Council Directive 67/548/EEC, 28th ATP), R48 should be applied with the following evidence:

(Those with specific relevance for the classification of haemolytic anaemia are underlined and examples were given.)

If a haemolytic substance induces one or more of the serious health effects listed as examples below within the critical range of doses, classification with R48 is 
warranted. It is sufficient for classification that only one of these criteria is fulfilled.

\section{Substance-related deaths;}

Example:

- Premature deaths in anaemic animals that are not limited to the first three days of treatment in the repeated dose study. (Mortality during days $0-3$ may be relevant for acute toxicity.)

2. Major functional changes in organ systems (for example the lung);

Example:

- Clinical signs of hypoxia, e.g. cyanosis, dyspnoea, pallor,... in anaemic animals that are not limited to the first three days of treatment in the repeated dose study.

3. Any consistent changes in clinical biochemistry, haematology or urinalysis parameters which indicate severe organ dysfunction. Haematological disturbances are considered to be of particularly importance if the evidence suggest that they are due to decreased bone marrow production of blood cells;

Examples:

- Reduction in $\mathrm{Hb}$ at $\geqslant 20 \%$.

- Reduction in functional $\mathrm{Hb}$ at $\geqslant 20 \%$ due to a combination of $\mathrm{Hb}$ reduction and MetHb increase.

- Haemoglobinuria that is not limited to the first three days of treatment in the repeated dose study in combination with other changes indicating significant haemolytic anaemia (e.g. a reduction in $\mathrm{Hb}$ at $\geqslant 10 \%$ ).

- Haemosiderinuria supported by relevant histopathological findings in the kidney in combination with other changes indicating significant haemolytic anaemia (e.g. a reduction in $\mathrm{Hb}$ at $\geqslant 10 \%)$.

4. Severe organ damage noted on microscopic examination following autopsy:

4.1 Widespread or severe necrosis, fibrosis or granuloma formation in vital organs with regenerative capacity (e.g. liver);

Example:

- Widespread or severe fibrosis in the spleen, liver or kidney.

4.2 Severe morphological changes that are potentially reversible but are clear evidence of marked organ dysfunction (e.g. severe fatty change in the liver, severe acute tubular nephrosis in the kidney, ulcerative gastritis); or

Example: 
- Severe tubular nephrosis

4.3 Evidence of appreciable cell death in vital organs incapable of regeneration (e.g. fibrosis of the myocardium or dying back of a nerve) or in stem cell populations (e.g. aplasia or hypoplasia of the bone marrow).

In the case where multiple effects of less severe grades in organs with regenerative capacity were obvious, the classification with R48 should also be applied because of the following criteria:

It is also important to consider not only specific severe changes in a single organ or biological system but also generalised changes of a less severe nature involving several organs or severe changes in general health status.

Example:

- Marked increase of haemosiderosis in the spleen, liver or kidney in combination with other changes indicating significant haemolytic anaemia (e.g. a reduction in $\mathrm{Hb}$ at $\geqslant 10 \%$ ) in a 28 day study.

- Significant increase in haemosiderosis in the spleen, liver or kidney in combination with microscopic effects like necrosis, fibrosis or cirrhosis.

Evidence indicating that $R 48$ should not be applied.

The use of this risk phrase is restricted to 'serious damage to health by prolonged exposure.' A number of substance-related effects may be observed in both humans and animals that would not justify the use of R48. These effects are relevant when attempting to determine a no-effect level for a chemical substance. Examples of well documented changes which would not normally justify classification with R48, irrespective of their statistical significance, include:

(a) Clinical observations or changes in bodyweight gain, food consumption or water intake, which may have some toxicological importance but which do not, by themselves, indicate 'serious damage';

(b) Small changes in clinical biochemistry, haematology or urinalysis parameters which are of doubtful or minimal toxicological importance;

Example:

- Significant decrease in $\mathrm{Hb}$ without any other significant indicators of haemolytic anaemia.

- Minimal to slight increase in MetHb formation without any other indications of significant haemolytic anaemia.

(c) Changes in organ weights with no evidence of organ dysfunction;

(d) Adaptive responses (e.g. macrophage migration in the lung, liver hypertrophy and enzyme induction, hyperplastic responses to irritants). Local effects on the skin produced by repeated dermal application of a substance which are more appropriately classified with R38 'irritating to skin'; or

Example: 
- Only adaptive or compensating effects without significant signs of haemolytic anaemia.

(e) Where a species-specific mechanism of toxicity (e.g. specific metabolic pathways) has been demonstrated.

Another example:

An increase in haemosiderosis without any other indications of significant haemolytic anaemia.

${ }^{1}$ Recovery means to gain full recovery within the time frame set in the test guideline for the chosen type of study. 